International Journal of Trend in Scientific Research and Development (IJTSRD)

Volume: 3 | Issue: 2 | Jan-Feb 2019 Available Online: www.ijtsrd.com e-ISSN: 2456 - 6470

\title{
Modeling and Analysis of Energy Efficient Cellular Networks
}

\author{
Iram Masood Hamdani' ${ }^{1}$, Raj Kumar Jain ${ }^{2}$ \\ ${ }^{1}$ M.Tech Scholar, ${ }^{2}$ Assistant Professor \\ Department of Electronics \& Communication Engineering, \\ Arya Institue of Engineering \& Technology, Kukas, Rajasthan, India
}

\begin{abstract}
Energy consumption has become a primary concern in design and operation of wireless communication networks due to two main reasons- Environmental concerns and Cost. The next generation network systems will have to consider energy efficient designs in any aspect. The $5 \mathrm{G}$ network which is most awaited today though proposes better data rates but also speaks about energy efficiency in its agenda.
\end{abstract}

KEYWORDS: 5G, beam forming, MIMO

\section{INTRODUCTION}

$5 \mathrm{G}$ networks is the most awaited mobile technology development in the next 5 years. A lot of talks, proposals and ideas have been put forward in many conferences, congresses and events across the globe regarding the requirements and deployment of this technology. Nextgeneration cellular communication systems, or $5 \mathrm{G}$, will be assisted by technologies that produce significant improvements in cell throughput. In recent years, various studies have focused on massive multiple input multiple output (MIMO) systems, which are considered to play a significant role in coming $5 \mathrm{G}$ technology. The technology till date has mainly concentrated on the data rates. But, energy also becoming an important parameter for the human survival hence plays a vital role in the enhancement of technology. Realizing this, industries are working on moving to renewable energy sources like solar or wind for powering the base stations. The results are promising and seem to reduce the stress on non-renewable resources. Massive MIMO is another area where a lot of research is being concentrated on, wherein the number of antennas is very large when compared to the number of users that the base station is serving. As the antennas increase, the amount of power consumed will greatly reduce. It is definitely true that the initial cost of installation of Massive MIMO will be very expensive as the hardware requirement is very high when compared to the current number of antennas that the telecom industry is using. But the energy savings by using Massive MIMO yield great results in the long run. A broad study of different techniques for energy efficiency reveals that beamforming also plays a very crucial role. Beamforming though has been into existence for more than over a decade, continuous improvements in the methodology keeps it ahead of many other technologies used for the common goal.

\section{Problem Definition}

Currently operating wireless networks have been mainly designed and deployed to maximize user's performance and focus on throughput, data rates and reliability, while usually paying less attention to energy efficiency. The future designs of wireless networks need to consider energy efficiency, since it is now a priority of the ICT industry to attain energy efficiency gains. Seeing this, a new research discipline called green cellular networks, concentrating on environmental influences of cellular networks, has been formed and attracted many researchers. The term green is originally a nickname for the dedicated efforts to reduce unnecessary greenhouse gases like (CO2) emissions from industries. The problem statement is described in the following points.

D. As for analysis the architecture of 4G LTE Network is not based on energy efficient concept even if there was no traffic load the network still consumes energy.

$>$ To minimize the cost energy consumption should be minimum hence the need of perfect energy efficient network.

$>$ As for the environmental considerations are concerned we need energy efficient networks.

III. Methodology

This work will adopt a research methodology that combines the theory model with empirical evaluation and refinement of the proposed scheme on MATLAB simulation tool. MATLAB is a useful high-level development environment for systems which require mathematical modeling, numerical computations, data analysis, and optimization methods. This is because MATLAB consists of various toolboxes, specific components, and graphical design environment that help to model different applications and build custom models easier.

Cellular network takes Energy consumption (in watt or joule/s) (Cost) as input and gives us Data throughput (in bit/s) (Benefit) as output. In order to measure how cost and benefit are balancing each other, we define benefit cost ratio:

$$
\begin{gathered}
\text { Energy efficiency [bit/joule] }= \\
\text { Data throughput [bit } / \mathrm{s} / \mathrm{km} 2] \\
\text { Energy consumption }\left[\frac{10 u l e}{\mathrm{~s}} / \mathrm{km} 2\right]
\end{gathered}
$$

For, Energy efficiency to be high, Energy consumption should be low. Also, due to environmental conditions and energy being non-renewable, energy consumption should be low. Also, energy costs money. In $4 \mathrm{G}$ network, as traffic load increases, we go from a rather high initial Energy consumption to slightly higher one, and Energy efficiency for that reason has grown steadily as the traffic load increases. The reason for initial cost when we don't have any traffic is because of the Architecture of $4 \mathrm{G}$. We have designed $4 \mathrm{G}$ network in the way that when no traffic is there, still energy is consumed by the network. We want energy consumption 
International Journal of Trend in Scientific Research and Development (IJTSRD) @ www.ijtsrd.com eISSN: 2456-6470

to be linear with traffic load and Energy efficiency be roughly high all the time. Now to find Energy Efficient Network Design, we have used Energy Efficiency Optimization method. The optimization methods are described below:

1. Select network design variables; $M, K, \rho, \lambda, \tau$

2. Model throughput and energy consumption as functions of these variables.

3. Solve :

\section{Maximize $(M, K, \rho, \lambda, \tau)$ \\ Data throughput $(M, K, p, \lambda, \tau)$ \\ Dner Ey consumption $(M, K, p, \lambda, \tau)$}

\section{Optimization Variables:}

$\mathrm{M}=$ Number of Antennas

$\mathrm{K}=$ Number of Active Users

$\mathrm{P}=$ Transmitted power

$\lambda=$ Base station density

\section{MODELING DATA THROUGHPUT}

Data throughput $=$ K. $\left(1-\frac{\tau K}{\mathrm{u}}\right) \operatorname{Blog}_{2}(1+\operatorname{SINR})$

$\mathrm{K}=$ Multiplexed users

$\left(1-\frac{\pi R}{\mathrm{u}}\right)=$ Data fraction per frame

$\operatorname{Blog}_{2}(1+\operatorname{SINR})=$ Data rate per user

$$
\operatorname{SINR}=\frac{I M}{\left(K+\frac{B N_{0}}{\rho}\right)\left(1+\frac{2}{\tau(\alpha-2)}+\frac{B N_{0}}{\rho}\right)+\frac{2 K}{\alpha-2}\left(1+\frac{B N_{0}}{\rho}\right)+\frac{K}{\tau}\left(\frac{4}{(\alpha-2)^{2}}+\frac{1}{\alpha-1}\right)+\frac{M}{\tau(\alpha-1)}}
$$

Pathloss exponent: $\alpha$

\section{MODELING ENERGY CONSUMPTION}

It depends strongly on hardware.

Characterized by parameters: $\mu, C_{0,0}, C_{0,1}, C_{1,0}, C_{1,1}, \mathrm{~A}$

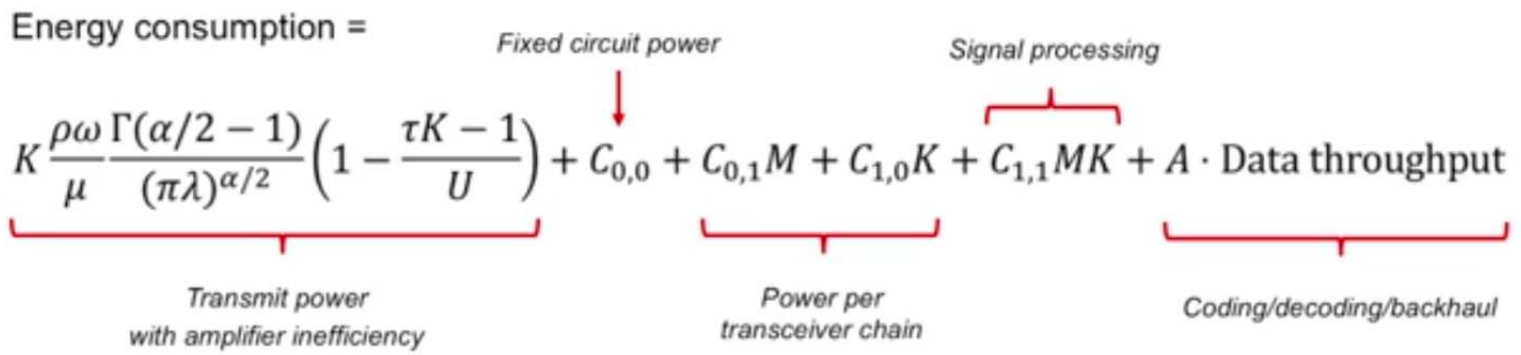

\section{Results}

The results are very important for research and development work to prove the problem definition practically. In my research I am using MATLAB tool to simulate the results.

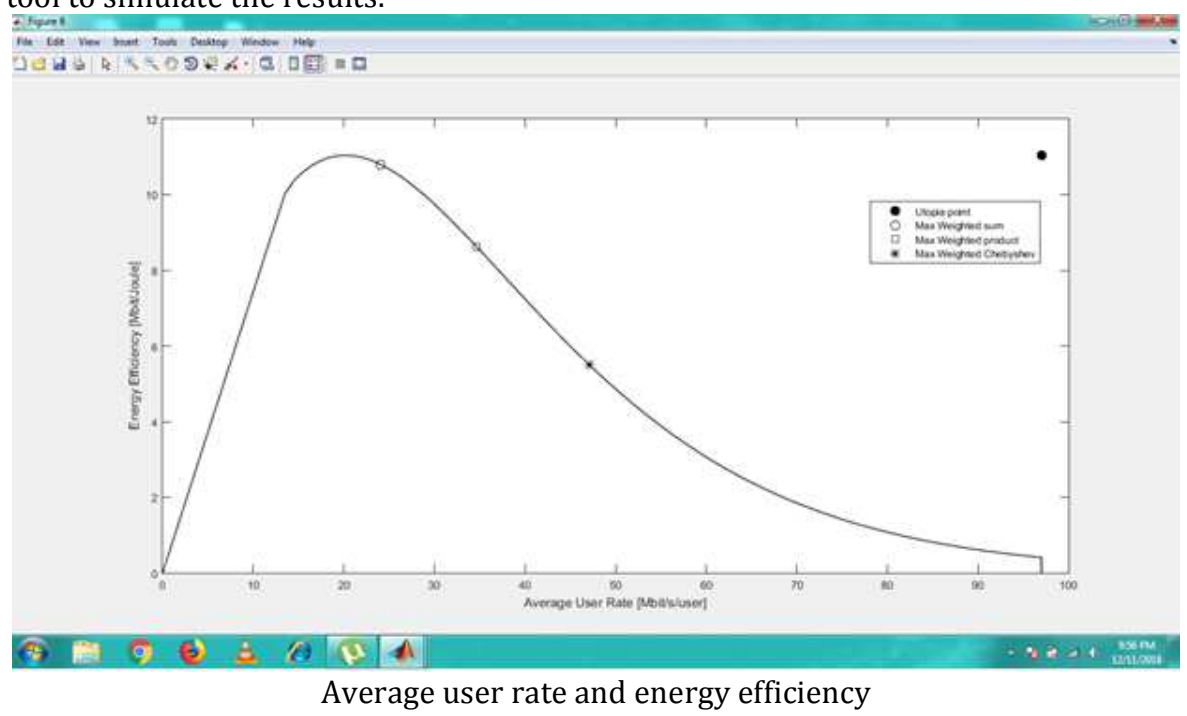



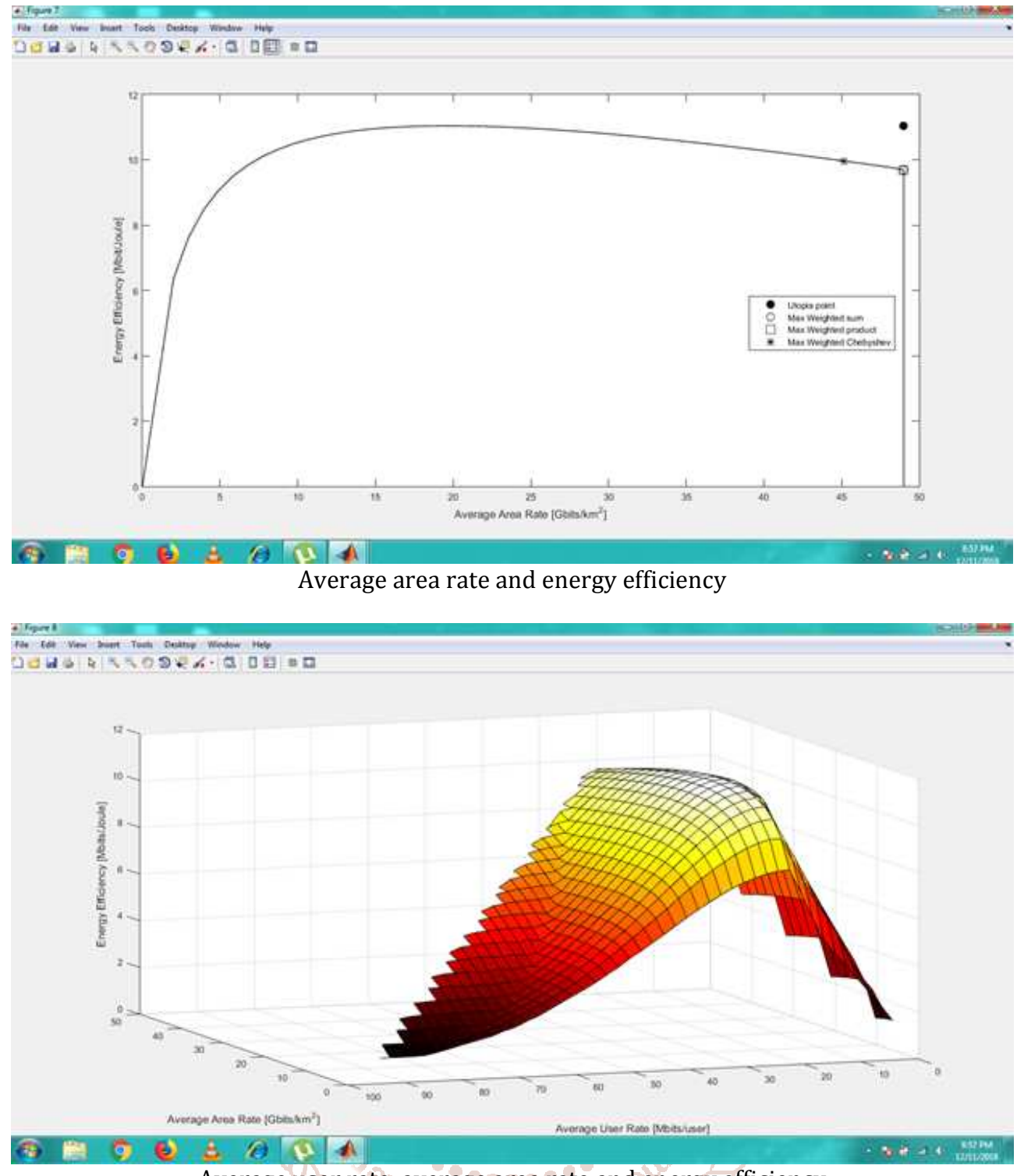

\section{Conclusion}

The aim of this paper is to present some of the most promising or potential modern wireless technologies capable in achieving positive energy savings over the existing methods, and to understand their potential and relations in energy savings. The work is an initial research towards achieving energy efficient $5 \mathrm{G}$ networks. A detailed initial study was done on the potential places where there is maximum power consumption in whole mobile network.

\section{References:}

[1] A. S. Andrae and T. Edler, "On global electricity usage of communicationtechnology: trends to 2030," Challenges, vol. 6, no. 1, pp. 117-157, 2015.

[2] MobiThinking, "Global mobile statistics." [Online]. Available: $\quad$ http://mobithinking.com/mobilemarketing-tools/latest-mobile-stats

[3] S. Vandris and K.-U. Killiches, "Power optimization in wireless heterogeneous networks," LSI White paper.

[4] G. Auer, V. Giannini, C. Desset, I. Godor, P. Skillermark, M. Olsson, M. A.Imran, D. Sabella, M. J. Gonzalez, 0. Blume et al., "How much energy isneeded to run a wireless network?" IEEE Wireless Communications, vol. 18,no. 5, 2011
[5] 0. Jumira and S. Zeadally, "Energy efficiency in cellular networks," EnergyEfficiency in Wireless Networks, pp. 1-16.

[6] J. M. Elmirghani, T. Klein, K. Hinton, T. El-Gorashi, A. Q. Lawey, andX. Dong, "Greentouch greenmeter core network power consumption modelsand results," in Green Communications (OnlineGreencomm), 2014 IEEE On-line Conference on. IEEE, 2014, pp. 1-8.

[7] O. Arnold, F. Richter, G. Fettweis, and O. Blume, "Power consumption modeling of different base station types in heterogeneous cellular networks," in FutureNetwork and Mobile Summit, 2010. IEEE, 2010, pp. 1-8.

[8] S. Cui, A. J. Goldsmith, and A. Bahai, "Energyconstrained modulation optimization," IEEE transactions on wireless communications, vol. 4, no. 5, pp.2349-2360, 2005.

[9] H. Holtkamp, G. Auer, S. Bazzi, and H. Haas, "Minimizing base station powerconsumption," IEEE Journal on Selected Areas in Communications, vol. 32,no. 2, pp. 297-306, 2014.

[10] R. Combes, S. E. Elayoubi, A. Ali, L. Saker, and T. Chahed, "Optimal onlinecontrol for sleep mode in green base 
International Journal of Trend in Scientific Research and Development (IJTSRD) @ www.ijtsrd.com eISSN: 2456-6470

stations," Computer Networks, vol. 78, pp.140-151, 2015.

[11] Z. Hasan, H. Boostanimehr, and V. K. Bhargava, "Green cellular networks: Asurvey, some research issues and challenges," IEEE Communications surveys \&tutorials, vol. 13, no. 4, pp. 524-540, 2011.

[12] T. Chen, H. Kim, and Y. Yang, "Energy efficiency metrics for green wirelesscommunications," in Wireless Communications and Signal Processing (WCSP),2010 International Conference on. IEEE, 2010, pp. 1-6.

[13] M. De Sanctis, E. Cianca, and V. Joshi, "Energy efficient wireless networkstowards green communications," Wireless Personal Communications, vol. 59,no. 3, pp. 537-552, 2011.

[14] H. Yang and T. L. Marzetta, "Total energy efficiency of cellular large scaleantenna system multiple access mobile networks," in Online Conference onGreen Communications (GreenCom), 2013 IEEE. IEEE, 2013, pp. 27-32.

[15] D. Feng, C. Jiang, G. Lim, L. J. Cimini, G. Feng, and G. Y. Li, "A survey ofenergy-efficient wireless communications," IEEE Communications Surveys \&Tutorials, vol. 15, no. 1, pp. 167-178, 2013.

[16] R. Fedrizzi, K. Gomez, S. Kandeepan, T. Rasheed, and C. V. Saradhi, "Energyaware routing in heterogeneous multi-hop public safety wireless networks," inCommunications Workshops (ICC), 2014 IEEE International Conference on.IEEE, 2014, pp. 218-224.

[17] S. Verdu', "Spectral efficiency in the wideband regime," IEEE Transactions onInformation Theory, vol. 48, no. 6 pp. 1319-1343, 2002.

[18] A. El Gamal, C. Nair, B. Prabhakar, E. Uysal-Biyikoglu, and S. Zahedi,"Energy-efficient scheduling of packet transmissions over wireless networks,"in INFOCOM 2002. Twenty-First Annual Joint Conference of the IEEE Computer and Communications Societies. Proceedings. IEEE, vol. 3. IEEE, 2002,pp. 1773-1782.।

[19] C.-Y. Li, C. Peng, S. Lu, and X. Wang, "Energy-based rate adaptation for802.11 n," in Proceedings of the 18th annual international conference on Mobilecomputing and networking. ACM, 2012, pp. 341-352.

[20] S. Nedevschi, L. Popa, G. Iannaccone, S. Ratnasamy, and D. Wetherall, "Reducing network energy consumption via sleeping and rate-adaptation." in NsDI,vol. 8, 2008, pp. 323-336.

[21] D. Xenakis, N. Passas, and C. Verikoukis, "A novel handover decision policy forreducing power transmissions in the two-tier lte network," in Communications(ICC), 2012 IEEE International Conference on. IEEE, 2012, pp. 1352-1356.

[22] Zheng Chang, Zhenyu Zhou, Sheng Zhou, Tao Chen, Tapani Ristaniem "Towards Service-Oriented 5G: Virtualizing the Networks for Everthing-as-a-Servive" 2017, IEEE Access.

[23] Ali Sahlli, Nordin, Mahamod Ismail, FadzilahAbdulah "Beamforming techniques for massive MIMO systems in 5G: overview, classification and trends for future research" 2017, Frontiers of Information Technology and Electronic Engineering, DOI: 10.1631/FITEE.1601817.

[24] G. Charis. N. Showme "Beamforming in Wireless Communication Standards: A Survey" 2017, Indian Journal of Science and Technology, Vol 10(5), DOI: 10.17485/ijst/2017/v10i5/99018

[25] Shoriful Islam, KartickMondal, TazkiaJessy "Suitable Beamforming Technique for 5G Wireless Communications" 2016, ResearchGate.

[26] Abdoulaye Tall, Zwi Altman,Eitan Altman "Multilevel beamforming for high data rate communication in $5 \mathrm{G}$ networks" 2015, arXiv:1504.00280v2 [cs.IT].

[27] WoonHau Chin, Zhong Fan, Russell Haines "Emerging Technologies and Research Challenges for $5 \mathrm{G}$ Wireless Networks"

[28] ZoranBojkovic, MiodragBakmaz, BojanBakmaz "On the Road to Energy Efficient 5G Mobile Networks" Recent Advances on Systems, Signals, Control, Communications and Computers.

[29] Arunitha, A., Gunasegaram, T., Kumar, N.S., et al., 2015. Adaptive beam forming algorithms for MIMO antenna. Int. J. Innov. Technol. Explor. Eng., 14(8):9-12.

Zaiyang Tang, Song Guo, Toshiaki Miyazaki, Hai Jin, Xiaofei Liao "Energy-Efficient Transmission Scheduling in Mobile Phones using Machine Learning and Participatory sensing" 2015, IEEE Transactions on Vehicular Technology, Vol. 64, No. 7.

[31] Navrati Saxena, Bharat J. R. Sahu, Young Shin Han "Traffic-Aware Energy Optimization in Green LTE Cellular Systems" 2014, IEEE Communications Letters, Vol. 18, No. 1.

[32] Levanen, Juho Pirskanen, Timo Koskela, Jukka Talvitie, Mikko Valkama "Radio Interface Evolution Towards 5G and Enhanced Local Area Communications" 2014, IEEE Access.

[33] Cheng-Xiang Wang, Fourat Haider, Xiqi Gao, Xiao-Hu You, Yang Yang, Dongfeng Yuan, Hadi M. Aggoune, Harald Haas, Simon Fletcher, Erol Hepsaydir "Cellular Architecture and Key Technologies for 5G Wireless Communication Networks" 2014, IEEE Communication Magazine.

[34] Yiqun Wu, Yan Chen, Jie Tang, Daniel K. C. So, Zhikun $\mathrm{Xu}$, Chih-Lin I, Paul Ferrand, Jean-Marie Gorce, ChihHsuan Tang, Pei-Rong Li, Kai-Ten Feng, Li-Chun Wang, Kai Börner, Lars Thiele "Green Transmission Technologies for Balancing the Energy Efficiency and Spectrum Efficiency Trade-off" 2014, IEEE Communication Magazine.

[35] K. Wieland, "mobileworldlive.com," GSMA, 8 May 2014. [Online].Available:http://www.mobileworldlive.com/. [Accessed 8 May 2014].

[36] “Wikipedia," Wikipedia, 12 January 2014. [Online]. Available:http://en.wikipedia.org/wiki/Directivity. [Accessed 2 May 2014].

[37] Emil Bjornson, Luca Sanguinetti, Jakob Hoydis, Merouane Debbah"Designing Multi-User MIMO for Energy Efficiency: When is Massive MIMO the Answer" 2013, 
International Journal of Trend in Scientific Research and Development (IJTSRD) @ www.ijtsrd.com eISSN: 2456-6470

https://www.researchgate.net/publication/25781160 3.

[38] Yao Chung "Novel Energy Efficient Transmission in 4G Downlink Networks" 2013, IEEE.

[39] Bechir Hamdaoui, Tamara Alshammari "Exploiting 4G Mobile User Cooperation for energy Conservation: Challenges and Opportunities" 2013, IEEE Wireless Communication.

[40] Jingqing Mei, Hong Ji, Yi Li “Energy Efficient Layered Broadcast/Multicast Mechanism in Green 4G wireless networks" 2011, IEEE INFOCOM 2011 Workshop on Green Communications and Networking.

[41] Burak Kantarci, Hussein T. Mouftah "Towards EnergyEfficient Hybrid Fiber-Wireless Access Networks" 2011, IEEE ICTON

[42] Sibel Tombaz, Anders Västberg, Jens Zander "EnergyAnd Cost -Efficient Ultra-High-Capacity Wireless Access" 2011, IEEE Wireless Communication.
[43] T. Haynes, A primer on Digital Beamforming, 1998.

[44] D. G. V. Tcheslavski, "ee.lamur.edu," 2008. [Online]. Available:ee.lamar.edu/gleb/em/Lecture $\% 2010 \% 20$ \%20Antennas.ppt.

[45] "Jordan University of Science and Technology," 02 Feb 2007.

[Online].

Available:www.just.edu.jo/ khodier/EE\%20700/ante nnas.pdf. [Accessed 10 Feb. 2014].

[46] S. J.Orfanidis, Electromagnetic Waves and Antennas, 1999-2010.

[47] T. Goncalves, T. U. o. L. L. P. IST, L. Correia and F. Cardoso, "Energy efficiency using beamforming at the base station in UMTS and LTE," in Software, Telecommunications and Computer Networks(SoftCOM), 2012 20th International Conference on, Split, 2012.

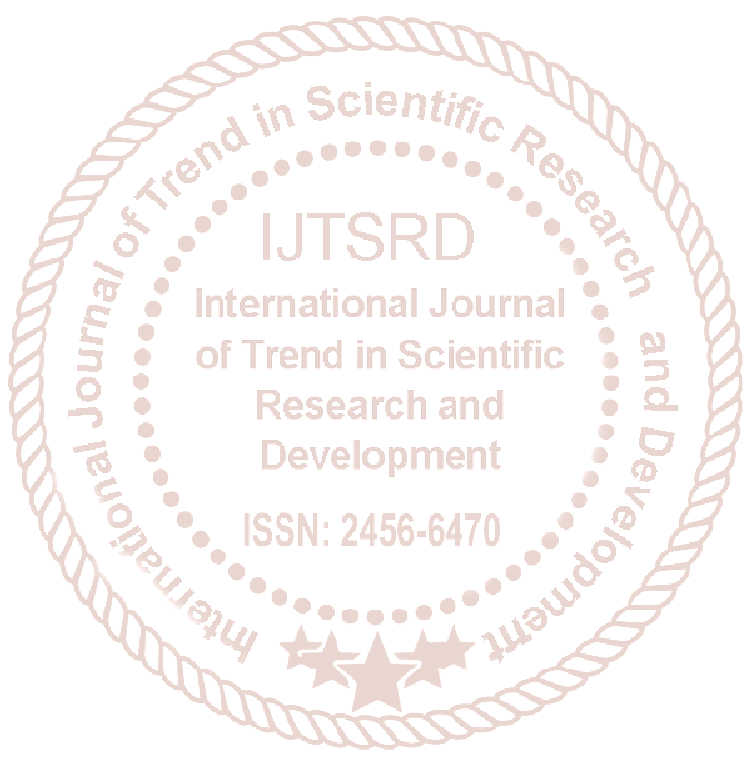

